\title{
Italique Italique
}

Poésie italienne de la Renaissance

XX $\mid 2017$

Varia

\section{Le Eclogae di Pontano e la bucolica in volgare di}

Sannazaro

\section{Vera Tufano}

\section{(2) OpenEdition}

1 Journals

\section{Edizione digitale}

URL: http://journals.openedition.org/italique/454

DOI: $10.4000 /$ italique.454

ISSN: 1663-4438

\section{Editore}

Librairie Droz

\section{Edizione cartacea}

Data di pubblicazione: 1 ottobre 2017

Paginazione: 73-94

ISBN: 978-2-600-05818-6

ISSN: 1423-3983

\section{Notizia bibliografica digitale}

Vera Tufano, "Le Eclogae di Pontano e la bucolica in volgare di Sannazaro », Italique [Online], XX | 2017, online dal 01 octobre 2019, consultato il 22 janvier 2020. URL : http://journals.openedition.org/ italique/454 ; DOI : 10.4000/italique.454

(c) Tous droits réservés 
Vera Tufano

LE ECLOGAE D I PONTA N O

E L A B UCOLICA I N V O G A E

D I S A N A Z A R 



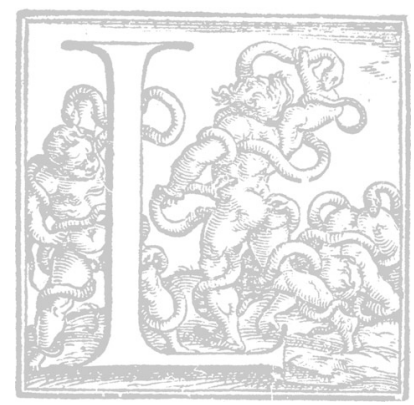

e Eclogae di G. Pontano rappresentano un prodotto letterario di grande interesse nel panorama della bucolica umanistica. Composte con tutta probabilità in momenti distinti della vita dell'umanista, conoscono una storia editoriale piuttosto tormentata. ${ }^{\mathrm{I}}$ Le prime quattro vengono inviate da Pontano stesso a Manuzio nel I503 (per comparire poi nell'editio princeps Aldina del I505). Di li a poco il poeta muore e nel I50s Summonte aggiunge alla raccolta di ecloghe altri due componimenti che appaiono nell'edizione napoletana delle prose pontaniane del I507. ${ }^{2} \mathrm{La}$ raccolta porta in sé le tracce di questa ripartizione, in quanto gli ultimi due componimenti appaiono 'estravaganti' rispetto agli altri quattro. La Monti Sabia ha dimostrato anche sulla base di testimonianze manoscritte che il quinto componimento, la Coryle, è il prodotto dell' assemblamento frettoloso, e non completamente rifinito, di due componimenti diversi, lavoro che probabilmente non fu completato per il sopraggiungere della morte del poeta. ${ }^{3}$

La raccolta è improntata a una spiccata eterogeneità e, in generale, presenta tratti fortemente sperimentali sia a livello contenutistico che linguistico-formale. Il primo componimento, la Lepidina, è un lunghissimo epitalamio per il matrimonio di Partenope e Sebeto, personificazioni rispettivamente della città di Napoli e del fiume Sebeto; interlocutori principali dell'ecloga sono Macrone e Lepidina, una coppia di sposi, probabilmente ispirata al poeta stesso e alla moglie, che recano doni pastorali alla coppia divina e che descrivono il corteo di invitati alle nozze che sfila per la città e che è composto da divinità che impersonano quartieri di Napoli e località del napoletano. ${ }^{4}$ Soggetto, estensione, stile, lessico avvicinino il componimento, oltre che alla bucolica, tanto al genere epitalamico, quanto all'epica e alla poesia d'amore elegiaca.

Il secondo componimento, il Meliseo, è un epicedio per la morte della moglie del poeta, Adriana Sassone, venuta a mancare nel I490, e trasfigurata nelle poesie del Pontano con il nome di Ariadna. Interlocutori sono $i$ pastori Cicerisco e Faburno che seguono da vicino il lutto di Meliseo, travestimento bucolico del poeta, nelle diverse fasi della sua rielaborazione. Il terzo componimento, il Maeon, è ancora un epicedio, questa volta per Paolo Attaldi, membro dell'Accademia Pontaniana, ma dai toni 
molto diversi rispetto alla seconda ecloga: più che un disperato coinvolgimento emotivo troviamo, nel dialogo fra i pastori Zefireo e Sincerio, una forte tensione speculativa su temi come la morte, la vita e il piacere. Il quarto componimento, Acon, vede ancora come protagonista Meliseo, alter ego pastorale del poeta, questa volta alle prese non più solo con la morte della moglie, ma con la serie di drammi che caratterizzarono l'ultima parte della vita dell'umanista.

La quinta ecloga, Coryle, presenta una prima parte in cui è narrato il mito di trasformazione dell'albero del nocciolo e una seconda parte, in distici elegiaci, in cui si racconta una favola con protagonisti Amore e Venere, e precisamente il rapimento di Amore da parte delle donne dei poeti d'amore latini come vendetta per le pene sofferte a causa dei loro uomini, sulla falsariga del Cupido cruciatus di Apuleio.

L'ultima ecloga, infine, il Quinquennius, è un mimo di grande tenerezza fra una mamma e il figlioletto di cinque anni, su temi della vita infantile e quotidiana familiare.

Questa rapidissima rassegna dei temi dei singoli componimenti fornisce già un'idea di base della ricchezza e multiformità di questa raccolta di ecloghe. E difficile individuare un elemento comune che tenga uniti $i$ diversi componimenti della raccolta e a volte, come nel caso dell'ultima ecloga, è difficile trovare elementi bucolici veri e propri. Il codice bucolico viene infatti approcciato con estrema disinvoltura e innovazione. ${ }^{7} L^{\prime}$ elemento bucolico prevalente potrebbe essere ravvisato proprio nella mascherata bucolica, nel travestimento bucolico che cela situazioni e personaggi propri del mondo del poeta.

La mascherata bucolica è, infatti, uno degli elementi costitutivi delle ecloghe del Pontano. La prima ecloga è caratterizzata da un pervasivo uso allegorico, per cui dietro al matrimonio di Partenope e Sebeto e la sfilata di eroi sono alluse una serie di circostanze reali quali le cerimonie che animavano la vita cittadina, come matrimoni regali, sfilate trionfali, cerimonie di consegna dei tributi. I due interlocutori principali, poi, sono al contempo identificabili come maschere bucoliche di due personaggi reali, il Pontano e la moglie, ma anche come emblemi poetologici di due tipi diversi di poesia: il poema epico (Macron) e la poesia d'ispirazione più raffinata e aggraziata, più lepida (Lepidina). ${ }^{8}$

Nella seconda ecloga due pastori riportano il lamento funebre di Meliseo per la morte della moglie Ariadna, in maniera simile alla XII ecloga dell'Arcadia di Sannazaro. L'identità di Meliseo e Ariadna è chiara; 
$i$ due interlocutori Cicerisco e Faburno banno nomi più oscuri, né nell'ecloga ci sono particolari che possono aiutare a identificarli meglio, a parte il fatto che si tratti di amici di Meliseo, a cui sta particolarmente a cuore la guarigione del pastore; con tutta evidenza si tratta di sodali dell'Accademia (tanto più se si vuole intravedere nell'ecloga e nella finale speranza che il pastore si prenda cura degli alberi, un invito a riprendere l'attività letteraria deposta a causa del lutto). Nell'ecloga dell'Arcadia $i$ due interlocutori sono Summonzio e Barcinio, vale a dire Summonte e Cariteo,' in mancanza di altri dati si può presupporre che $i$ due pastori del Meliseus di Pontano siano da identificare con gli stessi personaggi dell' Accademia.

Nella terza ecloga la mascherata bucolica riguarda ancora l' Accademia: il pastore Meone di cui si celebra la morte è il medico Paolo Attaldi dell'Accademia Pontaniana, dei pastori interlocutori, Sincerio e Zefireo, l'uno è da identificare con Sannazaro, l'altro è di difficile identificazione; la struttura del componimento farebbe pensare a una relazione di 'apprendistato' di Sincerio nei confronti dell'altro pastore; nella prima parte del componimento, Zefireo risponde alle domande di Sincerio, nella seconda, Zefireo dà avvio a un agone bucolico vero e proprio, in cui Sincerio-Sannazaro mostra un'abilità e un'eleganza pari a quella del suo interlocutore, ma forse minore scaltrezza e maggiore 'ingenuità'. Anche in questa ecloga, una delle meno conosciute, sembra di scorgere riflessi di temi ampiamente dibattuti nell'Accademia. Prima la riflessione sulla morte e sulla sopravvivenza o meno della vita, poi le immagini di piacere dei pastori, l'alternarsi delle stagioni, il rito funebre con l'addio al pastore sono temi presenti anche nell'Arcadia e sono usati qui per una riflessione sulla morte, sulla vita e sul piacere.

La quarta ecloga, l'Acon, mette in scena una mascherata bucolica il cui protagonista è ancora una volta Meliseo, e gli interlocutori due ortolani, Petasillo e Saliunco, che ne piangono l'assenza e ne ricordano icanti antichi d'amore e, quelli più recenti, di disperazione. Le figure dei due ortolani si celano sicuramente due sodali dell' Accademia, tuttavia i nomi delle maschere bucoliche in questo caso non sono trasparenti e si possono avanzare solo ipotesi; dall'ecloga, e in particolare dal finale, emerge l'immagine dei due ortolani come esponenti di un presente alternativo e meno glorioso rispetto all'epoca di grandezza che si sta concludendo; probabilmente le due figure rappresentano la generazione di accademici a cui Pontano, vecchio e provato da tragedie familiari e storiche, lascia il passo. 
La quinta ecloga, la Coryle, non presenta un andamento dialogico come le altre, ma destinatario è Azio, nome accademico di Sannazaro: a lui il poeta affida la pianta del nocciolo, da cui prende nome l'ecloga stessa, in una sorta di testamento letterario, in cui l'allievo prediletto diviene depositario dell'eredità poetica del maestro, ma soprattutto della sua cifra innovatrice.

Il dialogo fra la madre Pelvina e il figlio di cinque anni contenuto nella sesta ecloga pare invece adombrare un dialogo familiare fra la moglie del poeta e il figlio. ${ }^{\mathrm{I}}$

Nata in seno alla corte e all'accademia, la poesia delle Eclogae è specchio di orientamenti letterari ben precisi, che hanno esiti letterari coerenti e che attingono a un bagaglio di valori, gusti letterari e ideologici comune agli intellettuali che fanno parte di quel circolo culturale. Nella raccolta sono diversi i punti da cui emerge la consapevolezza dell'autore d'inserirsi in una linea di tradizione ben delineata, di cui si rappresenta un presente fulgido, che è all'altezza dell'illustre passato, ma ̀̀ anche capace di consegnare al futuro una valida continuità tramite $i$ suoi allievi.

La prima ecloga, la Lepidina, presenta una sezione finale occupata da diverse profezie relative alla stirpe che nascerà dalle nozze di Partenope e Sebeto $(723-81) ;{ }^{I}$ in questa parte il poeta ritaglia una profezia tutta per sé: dopo essersi collocato in successione immediata con Virgilio e aver tentato una sourapposizione fra le sue vicende biografiche e quelle del predecessore latino, ${ }^{\mathrm{I}}$ lascia intuire una prospettiva futura in questa 'catena' bucolica: altri pastori illustri seguiranno le sue orme e gli dedicheranno l'estremo saluto, riconoscendogli, in qualche modo, un ruolo di autorità. ${ }^{3}$ In questa proiezione futura si puì leggere con tutta probabilità un riferimento all'allievo Sannazaro.

In un passo della quinta ecloga, Pontano ripropone questa idea di successione, per cui la sua bucolica si inserisce in una tradizione dal glorioso passato. La voce narrante afferma di aver ascoltato il mito di trasformazione del nocciolo - inserito nella prima sezione dell'eclogadal vecchio Amilcone, che aveva avuto come maestro Titiro, alludendo poi velocemente a suoi successori («inde alii», "quindi sono venuti altri"). ${ }^{\mathrm{I}}$

Non sarà un caso che proprio la quinta ecloga, in cui è espressa questa dichiarazione di poetica, sia dedicata a Sannazaro, figura molto presente nella raccolta. L'incipit dell'ecloga presenta una sorta di passag- 
gio di consegna fra il maestro e l'allievo, a cui viene affidato la pianta del nocciolo, che assurge qui a simbolo della poesia del Pontano, una poesia bucolica alternativa rispetto alla tradizione, ma comunque altrettanto valida. ${ }^{\text {'s }}$ In questo incipit, che è molto denso dal punto di vista metaletterario, il riferimento al lutto di Meliseo allude alla seconda ecloga pontaniana, l'epicedio per Ariadna, che si può leggere in perfetto parallelismo con la dodicesima ecloga dell'Arcadia di Sannazaro. Tra $i$ due fronti bucolici, le Eclogae del Pontano e l'Arcadia, è dunque in atto un dialogo intimo e fitto, che è testimoniato da ulteriori parallelismi, celati in raffinatissime allusioni metaletterarie.

Nel prologo dell'Arcadia, lo stesso Sannazaro, dedicandosi ad analoghe dichiarazioni poetologiche, sembra inserirsi programmaticamente in questa tradizione quando afferma di voler raccontare "egloghe roz:e»", alla maniera in cui le ha sentite «sotto le dilettevoli ombre, al mormorio de liquidissimi fonti, da' pastori d'Arcadia». ${ }^{16}$ L'elemento più indicativo in questo passo è l'aggettivo liquidissimus; esso non compare nella poesia latina del Sannazaro, ma è presente anche nella quinta pompa della Lepidina ${ }^{17}$ - e nella poesia del Pontano solo in questo caso peraltro in un passaggio dallo spiccato valore metaletterario; durante la presentazione che la ninfa Planuride fa degli eroi dei monti che prendono parte al corteo nuziale, ${ }^{\mathrm{I}}$ la protagonista Lepidina constata la stanchezza della ninfa e la rassicura che le porterà dell'acqua attinta a un "fiume limpidissimo" (liquidissimus amnis) che scorre nelle vicinanze $(395-96) .{ }^{\text {I9 }}$

Il passaggio presenta un carattere metaletterario abbastanza scoperto: infatti, subito dopo la promessa delle coppe d'acqua, Lepidina indica a Planuride la presenza non troppo lontana della sorella Urania, definendola "sorella maggiore d'età". ${ }^{\circ}$ In questo particolare che sembra inserito quasi di fretta, il Pontano cela in verità un riferimento al suo poema Urania, opera 'sorella' della Lepidina, in quanto creata dallo stesso autore e affine nell'inventiva mitologica, ma "maggiore", in quanto appartenente a un genere "maggiore".

Non è difficile immaginare che un frangente che dà voce a una considerazione poetologica cosi scoperta, per quanto solo accennata, diventi a sua volta oggetto di allusione metaletteraria. Sannazaro afferma di aver ascoltato presso fonti "liquidissimi" egloghe rustiche; presso una fonte liquidissima la ninfa Planuride canta gli eroi della quinta pompa della Lepidina. Fra l'altro, in questo passaggio dell'ecloga pontaniana, il 
racconto di Planuride è relativo agli Orchi, divinità che impersonano $i$ Campi Flegrei, la cui raffigurazione si avvicina molto alla descrizione dei vulcani impersonati dai Giganti ribelli che fa la ninfa che accompagna Sincero nella catabasi della dodicesima prosa dell'Arcadia. Se nella Lepidina, Planuride fornisce una raffigurazione dei Campi Flegrei come mostri dagli occhi infuocati e dalle esalazioni sulfuree, ${ }^{2 \mathrm{I}}$ e non manca di ricordare il gigante Tifeo, ${ }^{22}$ nell'Arcadia allo stesso modo vengono presentati, tramite l'immagine del fuoco e l'odore dello zolfo, $i$ Giganti ribelli e viene evocata la figura del mostro Tifeo. ${ }^{23}$ Ed è proprio nella dodicesima prosa dell'Arcadia, nella sezione finale del suo viaggio, che l'autore usa l'espressione «liquidissimo fiume» per il Sebeto. ${ }^{24}$ Cosi come Lepidina allude a un fiume liquidissimus al termine della descrizione dei giganteschi Orchi dei Campi Flegrei fatta da Planuride, Sincero invoca Sebeto come «liquidissimo fiume» alla fine della sua discesa ctonia, in cui erano stati evocati $i$ Giganti giacenti sotto $i$ vulcani, con riferimento specifico ai Campi Flegrei. Ci troviamo quindi di fronte a una catena circolare di allusioni, richiami, corrispondenze, in una complessissima trama di scambi, convenzioni e riflessioni letterarie interna all' Accademia.

Un altro dei moduli ricorrenti tanto nelle ecloghe di Pontano quanto nella bucolica di Sannazaro è sicuramente quello del lutto per la morte di un pastore. La quinta prosa e la quinta ecloga dell'Arcadia sono dedicate alla morte del pastore Androgeo, di cui è descritto il rito funerario e a cui il pastore Ergasto dedica un canto commemorativo. ${ }^{25}$ Il lutto è uno dei motivi che appartengono al genere bucolico sin dalle sue origini piu antiche, a partire dalla rappresentazione della morte di Dafni, ${ }^{26}$ e sembra che nella bucolica napoletana rinascimentale venga recepito come una delle componenti essenziali del genere. In ragione di questa predilezione parla sicuramente il fatto che le ecloghe rappresentino in epoca rinascimentale una poesia d'occasione, attraverso la quale vengono scandite e celebrate le fasi principali della vita di corte e di accademia. Cosi come venivano scritte, e rappresentate, ecloghe in occasione di feste e avvenimenti lieti, quali nozze regali o celebrazioni di vittorie militari, allo stesso modo si ricorreva all'ecloga anche in occasione di ricorrenze funebri e morti illustri. ${ }^{27}$

Il rito funebre di Androgeo e la celebrazione del defunto da parte dei pastori sodali mostrano numerose analogie con la cerimonia funebre per la morte di Ariadna descritta nella seconda ecloga pontaniana, il 
Meliseus; entrambi $i$ riti sono celebrati alla maniera antica, con l'offerta sul tumulo di doni votivi come il latte e altri alimenti; ${ }^{28}$ inoltre la lunga serie di domande retoriche che accompagna la morte di Androgeo, ${ }^{29}$ con cui ci si chiede cosa resti al mondo dopo la dipartita del pastore, ricorda da vicino le martellanti domande retoriche e le disperate considerazioni del threnos per Ariadna nella seconda ecloga pontaniana. $^{30}$

Ma l'epicedio per Androgeo presenta ancora più affinità con l'epicedio per Paolo Attaldi della terza ecloga pontaniana, il Maeon. Protagonisti del componimento sono due pastori, Sincerio e Zefireo, di cui uno, Sincerio, è da identificare chiaramente con Sannazaro stesso. Lo schema dell'epicedio è simile nei due passaggi: alle riflessioni su cosa resti del defunto, segue la lode dell'amico e, infine, l'eterno saluto. Ma i due testi sembrano dialogare anche su un altro aspetto. Il pastore dell'Arcadia che conduce l'elogio di Androgeo rimane colpito dalla mancanza di sensi del defunto allo stesso modo del Sincerio del Maeon; nell'ecloga pontaniana l'argomento viene risolto con una visione del tutto atea e cerebrale della morte, ${ }^{3 \mathrm{I}}$ nell'Arcadia il pastore ugualmente coglie il contrasto fra la propria fede, che lo spinge a credere nell'immortalità dell'anima del sodale, e il vuoto creato dalla sua morte nella realtà di tutti i giorni, pur risolvendolo in senso cristiano. ${ }^{32}$

L'argomento non è di poco conto, in quanto il Maeon sembra riecheggiare questioni ampiamente dibattute nell'Accademia; dopo aver mostrato che cos'è la morte e aver ribadito con forza che nulla sopravvive a essa, $i$ due pastori pontaniani infatti si confrontano in un agone che ba come tema centrale il piacere e alterna scene pastorali dominate dalla voluptas. Quello sulla voluptas e sul bene umano da identificare in una vita dopo la morte, o al contrario nel piacere terreno, costituiva un dibattito che aveva infiammato la prima generazione di intellettuali aragonesi ed era costato caro a Lorenzo Valla, sulla cui visione epicurea aveva prevalso, alla fine, la soluzione cristiana di Facio: ${ }^{33}$ nella seconda generazione sembrano tuttavia riaffiorare lontani echi di questo dibattito che doveva essere ben noto agli esponenti dell'Accademia e poteva tranquillamente emergere in uno spazio letterario più aperto e 'dilatato' come quello della bucolica, tanto in latino quanto in volgare. Ancora una volta, quindi, la bucolica latina pontaniana e quella in volgare di Sannazaro mostrano stretti punti di contatto dettati da una comune matrice intellettuale e letteraria. 
Un altro modulo che accomuna la produzione bucolica di Pontano e quella di Sannazaro (tanto l'Arcadia quanto le egloghe piscatorie) è l'attenzione alla geografia della Campania, che ne fa una poesia molto sensibile al dato attuale e arriva a delineare una nuova geografia bucolica, che avrà molta fortuna negli esiti piscatori successivi, dove diventa topica la descrizione delle bellezze del golfo. ${ }^{34}$

Nella prosa XII dell'Arcadia Sannazaro-Sincero paga il suo tributo alla patria, nella narrazione del viaggio di catabasi e risalita di cui è stato protagonista. Non è un caso che in questa descrizione venga attribuito un ruolo centrale al Sebeto, definito «re del mio Paese» e di cui viene descritto con precisione l'andamento del corso. ${ }^{35}$ Nella Lepidina del Pontano, Sebeto è protagonista di nozze regali con Partenope ed è descritto, al pari della sposa, con attributi regali. ${ }^{36}$ Il Sebeto è, anzi, il grande protagonista della prima ecloga pontaniana, insieme a Partenope. Dietro a questa centralità del fiume, come succede in tutta la Lepidina, si nasconde un dato attuale di estrema importanza, la rivalorizzazione del fume in coincidenza con $i$ lavori di restauro $e$ sistematizzazione dell'approvvigionamento idrico della città che ebbero luogo negli anni'so che va di pari passo con un'operazione più ampia di decorazione architettonica della città, fortemente influenzata dall'ideologia aragonese. ${ }^{37}$ Punto focale del progetto di rinnovamento architettonico della città ad opera degli Aragonesi fu la realizzazione della villa di Poggioreale nel I487, sotto la cura del duca di Calabria Alfonso; la villa e il complesso dei suoi giardini erano riforniti di acqua da un sistema idrico incentrato sul corso del Sebeto, che andava cosi a rivestire un ruolo di primaria importanza nell'impianto ideologico aragonese. ${ }^{38}$ Se la Lepidina riflette in maniera perspicua questa realtà, nell'Arcadia ugualmente si possono cogliere echi di questo interesse, nell'attenzione e centralità riservata al Sebeto.

Questa sensibilità al dato attuale produce un'inevitabile dilatazione del codice bucolico. Lo spazio diventa quello napoletano e i codici letterari a cui si attinge per venire incontro a questa esigenza di innovazione si moltiplicano.

Uno dei dati che più colpisce nelle Eclogae del Pontano è il superamento di una dimensione bucolica classicamente intesa con l'apertura ad altri codici della tradizione, con l'inserimento disinvolto di elementi presi in prestito da altri spazi e codici, come quello georgico o elegiaco, o con la fondazione di una nuova dimensione, e un nuovo tipo di ecloga, 
l'ecloga holitoria. Questo processo si svolge, come consueto nel Pontano, con un alto grado di consapevolezza poetica e accompagnato da dichiarazioni celate dietro il velo dell' allegoria. Alcuni passi della bucolica in volgare di Sannazaro sembrano riflettere, allo stesso modo, questa nuova sensibilità.

Lo struggimento e la disperazione del pastore per un amore infelice rappresentano uno dei motivi ben noti alla tradizione bucolica, tuttavia è emblematica del nuovo modo di concepire il genere la soluzione che viene fornita in Pontano e nell'Arcadia. Il dolore del pastore Meliseo per la morte della donna è seguito nella sua evoluzione da Faburno e Cicerisco, $i$ due interlocutori della seconda ecloga: allinizio quello di Meliseo sembra un dolore senza via di uscita, destinato a lasciare il pastore nel silenzio e nell'ombra; verso la fine dell'ecloga, tuttavia, compaiono incoraggianti segnali di ripresa. I due interlocutori indicano la possibile via di uscita dal lutto e dallo stato di prostrazione in cui è caduto Meliseo nell'arboricoltura. ${ }^{39}$ Insitio, l'innesto, è la parola chiave della sezione: la cura degli alberi e la necessità del loro innesto richiameranno il poeta ai suoi orti abbandonati. Insitio è parola ovidiana e ai Remedia amoris dell'amato Ovidio allude sicuramente il Pontano nella sua bucolica che ha frequentissime aperture alla poesia elegiaca e alla poesia d'amore latina. ${ }^{40}$

Allo stesso modo, nell'ecloga VIII dell'Arcadia, Eugenio cerca di consolare l'amico Clonico dolente per amore e il rimedio alla sua disperazione è additato nella cura degli alberi, che deve distogliere il pastore dall'ozio, inteso catullianamente come condizione molesta per l'amante. ${ }^{4 \mathrm{I}}$ C'è di più. Il finale della seconda ecloga di Pontano si chiude programmaticamente con la parola libro, ${ }^{42}$ che ha un doppio livello di interpretazione: può essere intesa come la corteccia degli alberi di cui deve tornare a prendersi cura Meliseo, ma può intendersi anche nell'accezione di libro vero e proprio, e fare riferimento a un'opera a cui il poeta deve tornare a dedicarsi dopo l'interruzione causata dal dolore per la perdita della donna amata. La mia opinione è che si faccia qui allusione al De hortis Hesperidum, il poema didascalico che Pontano compone alla maniera georgica sulla cura dei cedri, e in questa direzione potrebbero essere indicativi anche $i$ diversi riferimenti all'albero contenuti nell'ecloga. ${ }^{43}$ Numerose suggestioni letterarie si intrecciano nell'ecloga pontaniana, che si apre e confonde con molteplici codici, cosi come nella bucolica di Sannazaro. D'altra parte, nella 
seconda ecloga pontaniana lo stesso Meliseo era stato raffigurato, insieme alla moglie, più come un contadino, dedito ai lavori dei campi, che come un pastore. La soluzione che è data al suo dolore è una soluzione addirittura elegiaca. E il libro a cui si allude allegoricamente è un'opera didascalica sulla coltivazione dei cedri. I confini già labili del genere si sfaldano sempre di più con la bucolica umanistica. I pastori di queste ecloghe non sono più rigorosamente pastori, perché molteplici sono diventate le istanze poetiche che gli intellettuali dell'Accademia pontaniana affidano alla bucolica.

Alla dimensione spaziale bucolica si affiancano nelle ecloghe di Pontano almeno altre due dimensioni, che si ritrovano anche in Sannazaro: una dimensione georgico-olitoria e quella del mare. Il poeta appare ben consapevole di questa apertura: non a caso nella Lepidina dichiara di essere consitor di un orto proprio (758); nella quarta ecloga, l'Acon, questo processo sembra portato alle estreme conseguenze e la dimensione bucolica e quella alternativa ortolana arrivano ad affiancarsi (e frizionare) in maniera dichiarata. Interlocutori dell'ecloga sono due maschere bucoliche, Petasillo e Saliunco (di difficile identificazione) che di bucolico non hanno quasi nulla, in quanto si presentano apertamente come "ortolani" e riconoscono uno scarto rispetto a Meliseo, che è designato come nobile pastore. ${ }^{44}$ Tuttavia, il pastore è ricordato come 'intermediario' fra le due dimensioni, amico degli ortolani e frequentatore della loro mensa. ${ }^{45}$

L'ecloga presenta una struttura metadiegetica piuttosto articolata, caratterizzata da più livelli temporali: Meliseo appare come oggetto del ricordo dei due ortolani e del pastore sono riportati canti collocati in diversi momenti della sua biografia; Meliseo è lontano, assente; $i$ due ortolani ne portano avanti il ricordo ed esprimono il rimpianto di non sentire più $i$ suoi canti; a sua volta, però, anche i due ortolani sono proiettati su un piano narrativo diverso da quello della voce narrante, in quanto alla fine viene meno la cornice narrativa del dialogo fra ortolani e Saliunco e Petasillo sono ritratti mentre raccolgono asparagi e funghi, avviandosi verso una capanna sudicia, dove apprendono la cottura di una vivanda a base di ortaggi raccolti.

Questa scena chiude l'ecloga dopo che Petasillo ha riportato l'ultimo canto-pianto di Meliseo, in cui il pastore piange tragedie familiari e politiche (la perdita della moglie, dei figli, la discesa dei Francesi, l'allontanamento dalle sue attività politiche e intellettuali): ${ }^{46}$ il canto di 
Meliseo si risolve nell' immagine finale di completo stravolgimento, in cui il pastore e il suo mondo subiscono la furia di una tempesta di proporzioni immani. ${ }^{47}$ Questa tensione non trova una vera soluzione, quando il canto del pastore si interrompe, si esce dalla cornice narrativa e si rimane con l'immagine dei due ortolani che proseguono nelle loro umili attività, ${ }^{4}$ simbolo di un mondo e di una storia che vanno avanti nella loro inesorabilità lasciandosi alle spalle un passato e una gloria non replicabili.

La quarta ecloga di Pontano presenta una sourapposizione di significati e messaggi tale da farne uno dei componimenti più complessi e preziosi della produzione dell'umanista. Collocata alla fine del nucleo (originario) di quattro ecloghe inviato da Pontano a Manuzio, essa sembra occupare una posizione 'finale' anche all'interno della biografia del poeta; rappresenta, anzi, quasi un bilancio della sua vita, della sua poesia, della sua vita accademica: siamo di fronte alla fine del sogno arcadico di Meliseo, e alla fine del sogno aragonese.

La stessa idea di stravolgimento che domina parte dell'Acon è avvertibile anche in alcuni passi dell'Arcadia. Interlocutori della decima ecloga dell'Arcadia sono Selvaggio e Fronimo; Fronimo prende la parola con un biasimo dei poeti/pastori moderni:
Selvaggio, oggi i pastor più non ragionano de l'alme Muse, e più non pregian naccari, per che per ben cantar non si coronano. E sì del fango ognun s'asconde i zaccari, che tal più pute che ebuli e abrotano e par che odore più che ambrosia e baccari. ${ }^{49}$

Selvaggio risponde e riporta il lamento di Caracciolo che mette in mostra un mondo completamente stravolto, che ricorda lo sconvolgimento che troviamo nel finale della quarta ecloga pontaniana..$^{\circ}$ Non sarà forse un caso che in entrambi passi ritornano due divinità come Pale e Vertumno, per il resto assenti nella bucolica pontaniana.

Mutata è la stagione sentenżia Caracciolo per mezzo di Selvaggio e anche in questo caso la raffigurazione del mutamento trova posto in una sezione conclusiva. Lo schema presente nell' ecloga dell'Arcadia e quello dell'Acon sono simili: in entrambi $i$ casi viene riportato il cantolamento di un illustre pastore che è però assente e di cui viene pianta la lontananza. Anche con il lamento di Caracciolo ci troviamo a un punto 
di snodo, il passaggio di stagione o forse sarebbe meglio dire, un passaggio di epoca, di una crisi che sta colpendo il regno aragonese e che la bucolica è chiamata a testimoniare, sia sul versante in volgare che su quello in latino. Se si accetta la datazione di Vecce, che fa risalire l'ecloga al $1485,{ }^{51}$ ci troveremmo dinanzi ai primi inquietanti segnali di crisi nell'Arcadia, e alla testimonianza di una definitiva decadenza nell'Acon.

Emblematicamente, sia l'Arcadia che la quarta ecloga di Pontano (che chiudeva il nucleo originario di ecloghe inviato dal Pontano a Manuzio) si concludono tragicamente e mestamente: la bucolica, il genere umile, ma cosi pervasivo in epoca rinascimentale, si fa carico di dare voce alla fine di un'intera epoca, in una coerente sincronizzazione letteraria e ideologica che travalica lo strumento della lingua, per rivelarsi comune al latino e al volgare.

Cosa rimane allora di questa illustre stirpe di pastori e dei loro canti? La bucolica e l'insegnamento di Pontano e Sannazaro sopravvivranno effettivamente all' antico splendore sotto nuove forme, meno 'impegnate', ma piuttosto fortunate: della dilatazione del codice bucolico operato nel laboratorio dell' Accademia sarà recepita soprattutto l'attualizazione geografica e l'apertura alla dimensione del mare, che daranno vita a una prolifica produzione piscatoria nel Cinquecento, con gli esperimenti poetici di Berardino Rota, Paolo Regio e Giulio Cesare Capaccio. ${ }^{52}$ La bucolica umanistica napoletana aggiunge un tassello fondamentale nella catena della tradizione del genere e, una volta esaurita la sua linfa vitale con il cessare delle condizioni storico-culturali che ne avevano garantito il valore letterario, lascia il posto a una nuova stagione, con esiti alternativi, ma duraturi, proprio come pronosticato dal Gran Pontano nel finale della sua quarta ecloga.

Vera Tufano 
I. Per la storia editoriale delle ecloghe si rimanda a Iohannis Ioviani Pontani Carmina, a cura di B. Soldati, Firenze, G. Barbera, I902, pp. LX-LxxxiII; L. Monti Sabia, Una schermaglia editoriale tra Napoli e Venezia agli albori del sec. XVI, «Vichiana» 6, i969, pp. 319-36; Iohannis Ioviani Pontani Eclogae, testo critico, commento e traduzione di L. Monti Sabia, Napoli, Liguori, I973, Introduzione, pp. 7-2 I.

2. Le due ultime ecloghe, la Coryle e il Quinquennius, vengono pubblicate nel I 507 in appendice ai dialoghi Actius, Aegidius e Asinus, presso i tipi di Sigismondo Mayr.

3. Sulla tradizione manoscritta della Coryle e la sua datazione rimane fondamentale lo studio di L. Monti Sabia, Esegesi e preistoria del testo nella Coryle del Pontano, in «Rendiconti dell'Accademia di Archeologia, Lettere e Belle Arti di Napoli», 45, I 970, pp. I 59-204.

4. Una prima indagine sulla struttura formale della Lepidina è contenuta in: W. L. Grant, An eclogue of Giovanni Pontano, «Philological Quarterly», 36, I 957 , pp. 76-84; alla Lepidina è dedicata gran parte dello studio sulle Eclogae condotto da $\mathrm{H}$. Casanova-Robin in: G. Pontano, Éclogues, Étude introductive, traduction et notes de H. Casanova-Robin, Paris, Les Belles Lettres, 20 II, pp. LXIII-CCLXXXII; per un'analisi dell'ecloga nel contesto storico e politico dell'epoca e del suo rapporto con le fonti classiche: C.V. Tufano, La Lepidina di Giovanni Pontano e il suo rapporto con il sistema dei generi letterari fra tradizioni antiche e innovazioni umanistiche, in «Studi Rinascimentali», 9, 20I I, pp. 37-5 I.

5. Fra gli studi più recenti sul Meliseus si ricordano: H. Casanova-Robin «Lauri, este mei memores»: Mémoire de l'épouse défunte dans la Deuxième Eglogue de Pontano. Réflexions sur une poétique mnémonique in Ecritures latines de la mémoire de l'Antiquité au XVIe siècle cur. H. Casanova-Robin, P. Galand, Paris, Classiques Garnier, 2010 (Colloques, congrès et conférences sur la Renaissance européenne 66) pp. 474, 327-59; C. V. Tufano, Tristitiae quoque meta sua est. Felicità e infelicità dei pastori pontaniani, in "Il barlume che vacilla". La felicità nella letteratura italiana dal Quattro al Novecento, a cura di V. Caputo, Milano, Franco Angeli, 2016, pp. I 5-35.

6. Per una trattazione delle peculiarità dell'epicedio della terza ecloga pontaniana vd. Tufano, Tristitiae quoque meta sua est. Felicità e infelicità dei pastori pontaniani, cit.

7. Sulla dilatazione degli usi e delle funzioni della bucolica in epoca rinascimentale si segnala il volume: D. Nelting, Frübneuzeitliche Pluralisierung im Spiegel italienischer Bukolik, Tübingen, Günter Narr, 2007 ("Romanica Monacensia" 74).

8. Per questa lettura meta-letteraria della prima ecloga vd. Tufano, La Lepidina di Giovanni Pontano, cit.

9. Per un'interpretazione dell'ecloga e dei suoi personaggi si rimanda a: IAcopo SAnnazaro, Arcadia, Introduzione e commento di C. Vecce, Roma, Carocci, 2013 , p. 324 .

I o. Una trattazione della sesta ecloga è presente in N. Thurn, Lateinischer Zugang. 23 lateinische Gedichte aus 24.Jabrbunderten. Übersetzt und besprochen, Rahden/Westf., Itinera Classica, 20I 4, pp. 224-55. 
I I. Per la sovrapposizione fra la figura di Virgilio e quella di Pontano e il tentativo di fornire una trasfigurazione napoletana alla figura virgiliana cfr. L. Monti Sabia, Trasfigurazione di Virgilio nella poesia del Pontano, in Atti del Convegno Virgiliano di Brindisi nel Bimillenario della Morte. Brindisi, I5-I8 ottobre 1981, Napoli, Liguori, I983, pp. 47-63.

I 2. Cfr. Pontano, Lepidina 757-66: «Nasceturque alius longo post tempore pastor / Aduena et ipse quidem, proprii sed consitor horti; / Ausit et hic tenerum calamo triuisse labellum. / Hunc et Damoetas et amabit Lyctius Aegon, / Alter oues niueas dono dabit, alter et hedos. / Hic pascet niueos herbosa ad flumina cygnos, / Misceat ipsa suos pascenti Amaryllis olores; / Hic et populea uacuus cantabit in umbra, / Vranie intactam cantanti iunget auenam / Et cantum argutae referent ad sidera ualles» ('Antiniana: Dopo lunghi secoli nascerà un altro pastore, straniero anche lui, ma coltiverà da sé un proprio orto ed oserà anche lui logorarsi le giovani labbra sulla zampogna. L'ameranno Dameta ed il lictio Egone, l'uno gli donerà pecore bianche come la neve, e l'altro dei capretti. Egli guiderà al pascolo candidi cigni lungo i fiumi erbosi ed Amarillide stessa verrà a mescolare ad essi i suoi cigni, ed egli canterà ozioso, disteso all'ombra di un pioppo, ed Urania unirà a quel canto il suono della sua vergine zampogna e le valli echeggianti rimanderanno quel canto fino alle stelle'). Il testo latino e la traduzione italiana sono tratti, qui e in tutti gli altri passi delle Eclogae che saranno citati, dall'edizione di L. Monti Sabia, Pontani Eclogae.

i 3. Pontano, Ecl. i, 769-75: «Succedentque alii Damones et Alphesiboei, / Quique etiam tenui musam meditentur auena / Pastores edera insignes et arundine clari. / O mihi tum ut choreas agitare et dicere uersus / Compositique senis mutae applausisse fauillae, / Vt iuuet et notam tumulo instaurare querelam: / Ipse senex tacita positus laetabitur urna» ('Antiniana: E nasceranno dopo di lui altri Damoni ed Alfesibei, e moduleranno anch'essi canzoni sul loro flauto sottile, pastori insigniti d'una corona d'edera e famosi per le loro melodie. Oh quanto mi piacerebbe allora poter danzare e recitare versi e plaudire al muto cenere del vecchio, ormai sepolto, e quanto ripetere sul tumulo il noto lamento: gioirà il mio vecchio, composto nel silenzio della sua tomba').

I4. Pontano, Ecl. 5, 22-3 I: «Sic olim puer audieram, dum cantat Amilcon / Ad choreas, nitet alba seni coma, deque galero / Cauda lupi et furuis horrent umbracula cirris. / Tityrus hunc docuit, sub quo caua fistula primum / Montibus his numeros deduxit et antra canore / Impleuit. Corydona quis aut non nouit Alexin, / Pastorum aut musam Damonis et Alphesiboei? / Inde alii. Imprimis bifori Corylenus auena / Saeuum arcu cassumque oculis et pectore Amorem / Obtrectare ausus, et amaro incessere cantu» ('Questa storia l'avevo sentita raccontare da ragazzo, mentre Amilcone cantava ad un ballo: splendeva al vecchio, tutta bianca, la chioma, e una coda di lupo gli pendeva giù dal berretto, la cui tesa era tutta irta di cirri neri. A lui fu maestro Titiro, al tempo del quale per la prima volta su questi monti il cavo zufolo sprigionò la sua melodia ed empì gli antri di canto: "Chi non conosce Coridone o Alessi, o la gara di canto dei pastori Damone ed Alfesibeo?" Dopo di lui sono venuti altri. Tra i primi Corileno, che sul suo zufolo con appena due buchi, ha osato rinfacciare ad Amore di essere spietato con l'arco e privo di occhi e di cuore, e sferzarlo con un sarcastico canto'). 
i 5 . Pontano, Ecl. 5, i-9: «Hanc, Acti, (neque enim patula solum esculus umbra / Grata placet) corylum tueare, nec arbutus una / Carmine nota dei est, Pana aut tegit una canentem, / Aestibus in mediis somnos suadente cicada; / Verum etiam et corylus nostris est cognita siluis, / Nec tantum Meliseus eam, aut tantum una Patulcis / Ornarunt calamis caesoque in cortice uersu, / Cum questu commota grauique excita querela / Vertice decuteret frondes» ('Abbi cura di questo nocciolo, o Azio: e infatti non è solo la quercia a piacere per la sua ombra, e non è solo il corbezzolo ad essere noto per il canto del dio, o a coprire con la sua ombra Pan che canta nel cuore dell'estate, mentre le cicale conciliano il sonno; ma anche il nocciolo è noto alle nostre selve, e non soltanto Meliseo o soltanto Patulcide l'hanno onorato coi loro zufoli e coi versi incisi nella corteccia, allorché, scosso dai singhiozzi e agitato da un sordo lamento, lasciava cader giù dalla sua cima le fronde').

i6. Sannazaro, Arcadia, Prologo, 4-5: «Dunque in ciò fidandomi, potrò ben io fra queste deserte piagge, agli ascoltanti alberi et a quei pochi pastori che vi saranno, racontare le rozze ecloghe da naturale vena uscite, così di ornamento ignude exprimendole come sotto le dilettevoli ombre, al mormorio de' liquidissimi fonti, da' pastori d'Arcadia le udii cantare; al le quali non una volta ma mille i montani idii da dolcezza vinti prestarono intente orecchie, e le tenere ninfe, dimenticate di perseguire i vaghi animali, lasciarono le faretre e gli archi appiè degli alti pini di Menalo e di Liceo». Per il passo dell'Arcadia qui riportato, e i successivi passi a cui si farà riferimento, si segue il testo di: SANnAZARO, Arcadia, Introduzione e commento di C. Vecce, cit.

17. La quinta pompa della Lepidina è costituita da un corteo di Orchi, personificazione dei Campi Flegrei, e «Oriarchi», eroi che impersonano diverse località montuose del napoletano, come il monte Gauro e il Vesuvio.

I 8. La ninfa Planuride è personificazione della località napoletana Pianura e a lei è affidata la descrizione della quinta pompa di eroi.

19. Pontano, Ecl. i, 395-96: «Lepidina: Et fessa es, mea Planuri, et liquidissimus amnis / It subter, sitienti et aquas et pocula promam» ('Tu sei stanca, Planuride mia, e proprio qui sotto scorre un fiume limpidissimo: tu hai sete, andrò a prenderti dell'acqua e dei bicchieri').

20. Pontano, Ecl. i, 397-99: «Proximaque Uranie scorteum et sua poma parauit, / Quae mihi cara soror forma prior et prior annis, / Quodque uides, summa procul innuit alta fenestra» (Qui vicino ha preparato un otre e i suoi bei frutti Urania, la mia cara sorella, che mi supera in età ed in bellezza e, come vedi, mi fa segno di lontano, lassù, dall'alto di quella finestra').

2 i. Pontano, Ecl. I, 374-83: «iter ingentes non explicat Orcos, / Quos acherusiacae fauces, noua numina, mittunt, / Stagnaque Baulorum, quos hostia pinguis Auerni / Emisere adytis lacus et fluitantis Araxi. / Mira illis sunt ora, soror, radiantia fronti / Lumina, sulfureis fumus de naribus efflat, / Tempora per serpunt rami mentoque rigescunt / Hircosae setae; tum guttura collaque circum / Squalent sulfureae totoque in pectore crustae, / Caetera membra nigror merus occupat et situs et nox» ('Il sentiero non basta ad incanalare gli Orchi immani, 
che, potenze sinora sconosciute, mandano fuori le fauci dell'Acheronte e le paludi di Bauli e che dagli abissi hanno vomitato fuori le porte del pingue Averno, e la fonte dell'Arasso ribollente. Spaventosi sono i loro volti, o sorella: ardono sulle fronti i loro occhi, dalle narici esalano vapori di zolfo, rami serpeggiano su per le loro tempie, sul loro mento si rizzano setole di capro. Oltre a ciò, tutto intorno al collo e alla gola e su tutto il petto, essi sono ricoperti di croste di zolfo; coprono il resto del corpo il nero più cupo e la muffa e la notte').

22. Pontano, Ecl. I, 388-91: «Ore latrant, saeuum ualeant qui inhibere Typhoea / Mouerat Aenaria ferus hic, et monte reuulso / Raptabatque iter et litus pede celsus obibat, / Intrabatque antrum» ('latrano in modo tale che paralizzerebbero di paura perfino il feroce Tifeo. Questo selvaggio gigante si era mosso, infatti, dall'isola Enaria e, divelta la montagna, divorava la strada e già, gigantesco come era, toccava col piede la riva del mare e s'avviava dentro la grotta').

23. Sannazaro, Arcadia, Prosa i 2, 27-30: «Così dicendo, cominciammo da lunge a scoprire un gran foco et a sentire un puzzo di solfo. Di che vedendo ella che io stava maravigliato, mi disse: "Le pene de' fulminati Giganti, che volsero assalire il cielo, son di questo cagione; i quali, oppressi da gravissime montagne, spirano ancora il celeste foco con che furono consumati. Onde adviene che, sì come in altre parti le caverne abondano di liquide acque, in queste ardeno sempre di vive fiamme [...] et appresso poi sotto la famosa Enaria, la quale voi mortali chiamate Ischia, ti mostrarei il furioso Tifeo, dal quale le estuanti acque di Baia e i vostri monti del solfo prendono il lor calore. Così ancora sotto il gran Vesevo ti farei sentire li spaventevoli muggiti del gigante Alcioneo; benché questi credo gli sentirai, quando ne advicinaremo al tuo Sebeto"».

24. Sannazaro, Arcadia, Prosa i 2, 40: «O liquidissimo fiume, o re del mio paese, o piacevole e grazioso Sebeto, che con le tue chiare e freddissime acque irrighi la mia bella patria, Dio ti exalte! Dio vi exalte, o ninfe, generosa progenie del vostro padre! Siate, prego, propizie al mio venire, e benigne et umane tra le vostre selve mi ricevete».

25. Sull'identificazione di Androgeo e di Ergasto si veda Sannazaro, Arcadia, p. I 34, in cui la figura di Ergasto è ricollegata a Sannazaro stesso e quella di Androgeo al padre di Sannazaro.

26. Una trattazione del motivo pastorale in questione, in particolare nei suoi esiti nella letteratura neo-latina è contenuta: B. Roling, Tod und Grabmal in der neulateinischen Eklogendichtung, in «Graeco-Latina Brunensia», 2009, I4, pp. 235-60.

27. Sull'uso dell'egloga in occasioni funerarie durante il Rinascimento: D. Ménager, L'églogue funèbre de la Renaissance in Les Funérailles à la Renaissance. XIIe colloque international de la Société française d'étude du Seizième Siècle, Bar-le-Duc, 2-5 décembre i999, ed. J. Balsamo, Genève, Droz, 2002, pp. 403-I 3.

28. In Sannazaro, Arcadia, Prosa 5, 21, i pastori offrono al defunto latte, sangue, vino e fiori: «E quivi (secondo lo antico costume) spargendo duo vasi di novo latte, duo di sacro sangue, e duo di fumoso e nobilissimo vino, e copia abundevole di tenerissimi fiori di diversi colori. et accordandosi con suave e pietoso 
modo al suono de la sampogna e de' naccari, cantava distesamente le lode del sepolto pastore». In Pontano, Ecl. 2, 98-ı I I, i doni offerti sul tumulo di Ariadna sono latte e miele: «Has lacrimas, Ariadna, atque haec tibi dona uouemus; / En lactis florem ad tumulum et redolentia mella, / Placamusque pios manes et condimus umbram, / Aeternum et ualeas, Ariadna, aeterna ualeto» ('queste lacrime, o Ariadna, e questi doni noi ti dedichiamo: eccoti sulla tomba il fior fiore del latte e il miele profumato e con essi plachiamo il tuo spirito pio e diamo pace alla tua ombra ed in eterno addio, Ariadna, addio in eterno!').

29. Sannazaro, Arcadia, Prosa 5, 24-27.

30. Pontano, Ecl. 2, 63-89; i 5 i-80.

3i. Pontano Ecl. 3, i-25: «Syncerius: Ipse uides, quo tot, Zephyree, inuenta sepulcrum / Cuncta tulit: superat uix, ah, uix est super umbra. / Zephyreus: Synceri, non umbra diu, non fama, nec ipsa / Exstabunt monumenta; rogo uix pauca supersunt; / Mox eadem nox obscura caligine condet, / Quae tumulo circum increscunt uirgulta uel alto / Ignea uis excussa polo, aut manus improba perdet, / Ossaque nuda solo sparsa atque ignota iacebunt; / Ne post ossa quidem, nec fabula Maeonis ulla» ('Sincerio: Lo vedi coi tuoi occhi, o Zefireo, il sepolcro dove tante scoperte tutte quante se l'è portate dietro: appena ne sopravvive, ahimè, appena ne avanza l'ombra! Zefireo: Non l'ombra, non la fama, e neppure gli stessi ricordi dureranno a lungo, o Sincerio; ben poco sopravvive alla morte, e presto anche questo poco lo seppellirà la notte nella sua fonda tenebra. I virgulti che spuntano intorno alla tomba o un fulmine scagliato giù dall'alto del cielo oppure una mano impietosa verrà a distruggerli, e le ossa, scoperte, giaceranno al suolo disperse e sconosciute; poi, neppure le ossa, neppure il ricordo di Meone').

32. Sannazaro, Arcadia, Prosa 5, 22-23: «Godi, godi, Androgeo, e se dopo la morte a le quiete anime è concesso il sentire, ascolta le parole nostre; e i solenni onori, i quali ora i tuoi bifolci ti rendono, ovunque felicemente dimori benigno prendi et accetta. Certo io creggio che la tua graziosa anima vada ora a torno a queste selve volando, e veda e senta puntalmente ciò che per noi oggi in sua ricordazione si fa sovra la nova sepultura. La qual cosa se è pur vera, or come può egli essere che a tanto chiamare non ne risponda?».

33. C.V. Tufano, Il De bumanae vitae felicitate di Bartolomeo Facio: tra modelli classici e fonti patristiche, in «Mélanges de l'Ecole française de Rome - Moyen Âge», I 28, I, 2016.

34. Sulla descrizione topica delle bellezze del Golfo di Napoli nella letteratura del Cinquecento di area napoletana: A. Mauriello, La Mergellina, nella poesia napoletana del secondo Cinquecento, in «Rinascimento meridionale», II, 20 I I, pp. I 57-70.

35. Sannazaro, Arcadia, Prosa xil, 40-4I.

36. Pontano, Ecl. i, 455-60; 71 7-i 8. 
37. Per il rinnovamento architettonico-artistico e il recupero dell'iconografia di Partenope nella Napoli aragonese: G. L. Hersey, Alfonso II and the Artistic Renewal of Naples 1485-1495, New Haven-London, Yale U.P., i 969; A. Beyer, Parthenope. Neapel und der Süden in der Renaissance, München-Berlin, Deut. Kunstverlag, 2000.

38. Sulla villa di Poggioreale si segnala l'importante volume di P. Modesti, Le Delizie ritrovate. Poggioreale e la villa del Rinascimento nella Napoli aragonese, Firenze, Olschki, 20I4.

39. Pontano, Ecl. 2, 242-45: «Faburnus: Tristitiae quoque meta sua est. Meliseus $\mathrm{ab}$ antro / Prodibit tandem segetis memor et memor horti, / Diluet et rastris curas et falce dolorem. / Ciceriscus: Quin potius, quoniam ver appetit et sua curae est / Insitio, falcemque illi cuneosque paramus? / Cortice quoque etiam lentescat vulnus et hudo, / Quae super ipse linens imponat glutina, libro» ('Faburno: Anche la tristezza ha la sua fine: Meliseo uscirà un bel giorno dall'antro, memore della messe e memore del suo giardino, e col rastrello allevierà gli affanni e con la falce il dolore. Cicerisco: Perché piuttosto, dal momento che la primavera si avvicina e gli sta a cuore l'innesto, non gli prepariamo la roncola ed i cunei? Anche così potrebbe rimarginarsi la sua ferita: maneggiando la corteccia e l'umido libro, su cui egli spalmi poi una mano di colla').

40. Sannazaro, Arcadia, Ecl. 8, i i 8-35: «Allora il nostro Pan colmo di grazia / con l'alma Pale aumenterà 'l tuo numero, / tal che la mente tua ne fia ben sazia. / E non ti sdegnerai portar su l'umero / la cara zappa, e pianterai la neputa, / l'asparago, l'aneto e 'l bel cucumero. / E 'l tempo sol in ciò disponi e deputa; / ché non s'acquista libertà per piangere, / e tanto è miser l'uom, quant'ei si reputa. / E poi cominciarai col rastro a frangere / la dura terra, e sterperai la lappola, / che le crescenti biade suol tant'angere. / Io con la rete ucello e con la trappola, / per non marcir ne l'ocio, e tendo insidie / a la mal nata volpe, e spesso incappola. / Così si scaccia amor; così le invidie / de' pastor neghittosi si postergano; / così si spregia il mondo e sue perfidie. / Così convien c'al tutto si dispergano / l'amorose speranze ardite et avide, / che ne le menti semplicette albergano».

4I. Nei Remedia amoris i lavori della campagna sono indicati come rimedio al mal d'amore e l'innesto viene evocato come attività che apre una nuova stagione in cui l'animo, intento nelle attività della campagna, scaccerà il pensiero dell'amore. Cfr. Ovid. Rem. i95.

42. Pontano Ecl. 2, 247-48: «Cortice quoque etiam lentescat uulnus et hudo, / Quae super ipse linens imponat glutina, libro» ('anche così potrebbe rimarginarsi la sua ferita: maneggiando la corteccia e l'umido libro, su cui egli spalmi poi una mano di colla').

43. I cedri sono testimoni del dolore di Meliseo: presso i cedri si innalza il lamento di Meliseo: Pontano, Ecl. 2, i 89-92: «Nuper et ad ueteres citrios, dum tondet anethum / Vxor et ipse simul mentam atque sisimbria purgo, / Suspirantem illum et querula cum uoce ferentem / Intenti accipimus» ('e poco fa, presso quei vecchi cedri, mentre mia moglie tagliava l'aneto ed io con lei ripulivo la menta e il sisimbrio, assorti nel nostro lavoro, lo sentiamo sospirare'); i cedri ne portano inciso il pianto: cfr. PONTANO, Ecl. 2, 202: «Triste gemunt scriptoque 
dolent in cortice cedri» ('i cedri piangono il lamento inciso nella loro corteccia’).

44. Pontano, Ecl. 4, 20-26: «Saliuncus: Non puduit cantorem igitur resonantis auenae / Cui tot oues, pecoris tantum, mulctralia tanta, / Pellitum pastorem olitorum accumbere mensis / Ulpicaque et ueteres ruptare ad prandia cepas. / Petasillus: Illum cepa recens uiridisque cucurbita captum / Ducebat mentaeque sapor succusque sisimbrii / Munere quo viridi recubans in cespite mecum» ('Saliunco: Dunque non ritenne vergognoso, il cantore dalla risonante zampogna, che pure aveva tante pecore, tante greggi, tanti vasi da mungere, sedersi, pastore ammantato di pelli, alla mensa degli ortolani e ruttare, a pranzo, porri e cipolle vecchie').

45. Per una lettura metaletteraria e un inquadramento storico-politico della quarta ecloga pontaniana vd. C.V. Tufano, Alcuni aspetti del lessico agro-alimentare nelle Eclogae di G. Pontano, in L'arte della parola e le parole della scienza. Tecniche di comunicazione nel mondo antico, Napoli, M. D'Auria Editore, 20I4, pp. 22 I-54.

46. Cfr. la lunga sezione finale dell'ecloga: PonTAno, Ecl. 4, i 40-78.

47. Pontano, Ecl. 4, I70-78: «Ah dolor, ah lacrimae, sonuere tonitrua coelo, / Discurrere faces, ignitaque tela coruscant, / De coelo tacti foetus, armenta gregesque / Et tactae uites auulsa et stiua serenti / Attonitu; uix ipse super turbatus et amens / Stragem inter pecorum frugumque relinquor, et atris / Ambustus flammis iaceo; uix colle propinquo / Lapsa Pales tutatur humi tollitque iacentem: / O mecum solitas ranae instaurate querelas / Et luctum geminate nouosque intendite questus» ('Ah sventura, ah pianto!, rimbombano i tuoni nel cielo, guizzano qua e là i lampi, balenano dardi di fuoco: sono colpiti dal fulmine i piccoli, gli armenti e le greggi e a me che semino viene strappato, in quel finimondo, il manico dell'aratro; a mala pena, stordito e sconvolto, sopravvivo in mezzo alla strage dell'armento e delle messi, e giaccio a terra, tutto annerito dalle fiamme; a mala pena dal colle vicino Pale, anche essa atterrata, mi protegge e mi aiuta a levarmi da terra. O rane, riprendete con me i vostri lamenti e raddoppiate il pianto e fate risuonare gemiti nuovi!').

48. Pontano, Ecl. 4, i 88-92: «Haec Saliuncus et haec referebat arundine clarus / Et calamis Petasillus, uterque insignis auena. / Interea asparagosque legunt fungumque recentem / Boletosque sequuntur et altercantur utrinque, / Conditura suo quae sit miscenda sapore» ('Così raccontava Saliunco, così Petasillo, famoso per il flauto e lo zufolo, entrambi egregi suonatori di zampogna. Frattanto vanno raccogliendo asparagi e funghi freschi, e vanno in cerca di boleti e discutono vivacemente tra di loro su quale condimento si debba mescolare al loro rispettivo sapore').

49. Sannazaro, Arcadia, Ecl. io, 4-9.

50. Sannazaro, Arcadia, Ecl. ıo, 8 5-92: «Mutata è la stagione e 'l tempo è duro, / e già s'attuffa Arcturo in mezzo l'onde; / e 'l sol, c'a noi s'asconde, ha i raggi spenti, / e van per l'aria i venti mormorando, / né so pur come o quando torne estate. / E le nubi spezzate fan gran suoni; / tanti baleni e tuoni han l'aria involta, / ch'io temo un'altra volta il mondo pera»; 98-г r 6: «O gloriosi spirti degli boschi; 
/ o Eco, o antri foschi, o chiare linfe, / o faretrate Ninfe, o agresti Pani, / o Satiri e Silvani, o Fauni e Driadi, / Naiadi et Amadriadi, o semidee, / Oreadi e Napee, or sète sole; / secche son le viole in ogni piaggia: / ogni fiera selvaggia, ogni ucelletto / che vi sgombrava il petto, or vi vien meno. / E 'l misero Sileno vecchiarello / non trova l'asinello ov'ei cavalca. / Dafni, Mopso e Menalca, oimè, son morti. / Priapo è fuor degli orti senza falce, / né genebro né salce è che 'l ricopra. / Vertunno non s'adopra in transformarse, / Pomona ha rotte e sparse le sue piante, / né vòl che le man sante puten legni. / E tu, Pale, ti sdegni per l’oltraggio, / ché di april né di maggio hai sacrificio».

5i. Sannazaro, Arcadia, p. 258.

52. Per un quadro d'insieme degli esiti 'piscatori' nella letteratura napoletana del Cinquecento fondamentale lo studio, già citato, di A. Mauriello, La Mergellina, nella poesia napoletana del secondo Cinquecento, cit.; sull'argomento si veda anche A. Mauriello, La Siracusa di Paolo Regio e la tradizione letteraria napoletana tra primo e secondo Cinquecento, in «Studi Rinascimentali», 6, 2008, pp. I-7, e sempre di A. Mauriello Il codice arcadico nella cultura napoletana del Cinquecento, in Iacopo Sannazaro. La cultura napoletana nell'Europa del Cinquecento. Atti del Convegno Internazionale di Studi (Napoli, 27-28 marzo 2006), a cura di P. Sabbatino, Firenze, Olschki, 2009, pp. 309-19. 